\title{
GRADIENT ESTIMATES AND HARNACK INEQUALITIES OF A PARABOLIC EQUATION UNDER GEOMETRIC FLOW
}

\author{
GUANGWEN ZHAO
}

\begin{abstract}
In this paper, we consider a manifold evolving by a general geometric flow and study parabolic equation

$$
\left(\Delta-q(x, t)-\partial_{t}\right) u(x, t)=A(u(x, t)), \quad(x, t) \in M \times[0, T] .
$$

We establish space-time gradient estimates for positive solutions and elliptic type gradient estimates for bounded positive solutions of this equation. By integrating the gradient estimates, we derive the corresponding Harnack inequalities. Finally, as applications, we give gradient estimates of some specific parabolic equations.
\end{abstract}

\section{INTRODUCTION}

The paper study parabolic equation

$$
\left(\Delta-q(x, t)-\partial_{t}\right) u(x, t)=A(u(x, t))
$$

on Riemannian manifold $M$ evolving by the geometric flow

$$
\frac{\partial}{\partial t} g(t)=2 h(t)
$$

where $(x, t) \in M \times[0, T], q(x, t)$ is a function on $M \times[0, T]$ of $C^{2}$ in $x$-variables and $C^{1}$ in $t$-variable, $A(u)$ is a function of $C^{2}$ in $u$, and $h(t)$ is a symmetric $(0,2)$-tensor field on $(M, g(t))$. A important example would be the case where $h(t)=-\operatorname{Ric}(t)$ and $g(t)$ is a solution of the Ricci flow introduced by R.S. Hamilton [12. We will give some gradient estimates and Harnack inequalities for positive solutions of equation (1.1).

The study of gradient estimates for parabolic equations originated with the work of P. Li and S.T. Yau [18. They prove a space-time gradient estimate for positive solutions of the heat equation on a complete manifold. By integrating the gradient estimate along a space-time path, a Harnack inequality was derived. Therefore, Li-Yau inequality is often called differential Harnack inequality. It is easy to see that the above space-time estimate will become an elliptic type gradient estimate for a time-independent solution (see [7]). But the elliptic type estimate cannot hold for a time-dependent solution in general, this can be seen from the form of the fundamental solution of the heat equation in $\mathbb{R}^{n}$. However, in 1993, R.S. Hamilton [14] established an elliptic type gradient estimate for positive solutions of the heat equation on compact manifolds. It is worth noting that the noncompact version of Hamilton's estimate is not true even for $\mathbb{R}^{n}$ (see [29, Remark 1.1]). Nevertheless, for complete noncompact manifolds, P. Souplet and Q.S. Zhang [29] obtained an elliptic type gradient estimate for a bounded positive solution of the heat equation after inserting a necessary logarithmic correction term. Li-Yau type and Hamilton-Souplet-Zhang type gradient estimates have been obtained for other nonlinear equations on manifolds, see for example [4, 5, 9, 17, 19, 23, 24, 27, 31, 33, and the references therein.

On the other hand, gradient estimates are very powerful tools in geometric analysis. For instance, R.S. Hamilton 13, 15, established differential Harnack inequalities for the Ricci flow and the mean curvature flow. These results have important applications in the singularity analysis. Over the past two decades, many authors used similar techniques to prove gradient estimates and Harnack inequalities for geometric flows. The list of relevant references includes but is not limited to [1, 3, 11, 16, 20, 22, 25,

2010 Mathematics Subject Classification. Primary 53C44; Secondary 35K55, 53C21.

Keywords. Parabolic equation, Gradient estimate, Harnack inequality, Geometric flow. 
30, 34, 35]. In this paper, we follow the work of J. Sun [30] and M. Bailesteanu et al. [1], and focus on the system (1.1) $-(1.2)$.

Now we give some remarks on equation (1.1). When $A(u)=a u \log u$, the nonlinear elliptic equation corresponding to (1.1) is related to the gradient Ricci soliton. When $A(u)=a u^{\beta}$, the nonlinear elliptic equation corresponding to (1.1) is related to the Yamabe-type equation. In general, the parabolic equation (1.1) is the so-called reaction-diffusion equation, which can be found in many mathematical models in physics, chemistry and biology (see 26, 28), where $q u+A(u)$ and $\Delta u$ are the reaction term and the diffusion term, respectively. The reaction-diffusion equations are very important objects in pure and applied mathematics.

In 6, Q. Chen and the author studied the equation (1.1) with a convection term on a complete manifold with a fixed metric. Here, we establish some gradient estimates for positive solutions of (1.1) under geometric flow (1.2), which are richer and sharper than [6].

The rest of this paper is organized as follows.

In Section 2 we establish space-time gradient estimates for positive solution of (1.1). We firstly consider that $M$ is a complete noncompact manifold without boundary. A local and a global estimate were established, see Theorem 2.1 and Corollary 2.6. Next, the case that $M$ is closed is also deal with. In this case, inspired by [1, we obtain a sharper estimate than [30, Theorem 6], see Theorem 2.7. We also give the corresponding Harnack inequalities in the above two cases, see Corollary 2.10.

In Section 3, we consider the case that the solution is bounded, and establish elliptic type gradient estimates of local and global versions, see Theorem 3.1 and Corollary 3.5. The elliptic type Harnack inequality is also obtained, see Corollary 3.6.

Finally, in Section 4 we give some applications and explanations of these gradient estimates in some specific cases. For the case of $A(u)=a u \log u$ with $a \in \mathbb{R}$, we can derive the gradient estimate for positive solutions. In particular, we deal with the case that the manifold evolving by the Ricci flow, see Corollary 4.2, 4.3 4.4. For the case of $A(u)=a u^{\beta}$ with $a \in \mathbb{R}$ and $\beta \in(-\infty, 0] \cup[1,+\infty)$, we give the gradient estimate for bounded positive solutions, see Corollary $4.5,4.6$.

Throughout the paper, we denote by $n$ the dimension of the manifold $M$, and by $d(x, y, t)$ the geodesic distance between $x, y \in M$ under $g(t)$. When we say that $u(x, t)$ is a solution to the equation (1.1), we mean $u$ is a solution which is smooth in $x$-variables and $t$-variable. In addition, we have to give some notations for the convenience of writing. Let $f=\log u$ and $\hat{A}(f)=\frac{A(u)}{u}$. Then

$$
\hat{A}_{f}=A^{\prime}(u)-A(u) / u, \quad \hat{A}_{f f}=u A^{\prime \prime}(u)-A^{\prime}(u)+A(u) / u .
$$

For $u>0$ we define several nonnegative real numbers (some of $\lambda, \Lambda, \Sigma, \kappa$ are allowed to be infinite) as follows:

$$
\begin{aligned}
& \lambda_{2 R}:=-\min _{Q_{2 R}, T} \hat{A}_{f}^{-}=-\min \left\{0, \min _{Q_{2 R, T}}\left(A^{\prime}(u)-A(u) / u\right)\right\}, \\
& \Lambda_{2 R}:=\max _{Q_{2 R, T}} \hat{A}_{f}^{+}=\max \left\{0, \max _{Q_{2 R, T}}\left(A^{\prime}(u)-A(u) / u\right)\right\}, \\
& \Sigma_{2 R}:=\max _{Q_{2 R, T}} \hat{A}_{f f}^{+}=\max \left\{0, \max _{Q_{2 R, T}}\left(u A^{\prime \prime}(u)-A^{\prime}(u)+A(u) / u\right)\right\} \\
& \kappa_{2 R}:=-\min \left\{0, \min _{Q_{2 R, T}}\left(A^{\prime}(u)-A(u) / u\right), \min _{Q_{2 R, T}} A^{\prime}(u)\right\}
\end{aligned}
$$


and

$$
\begin{aligned}
& \lambda:=-\inf _{M \times[0, T]} \hat{A}_{f}^{-}=-\min \left\{0, \inf _{M \times[0, T]}\left(A^{\prime}(u)-A(u) / u\right)\right\}, \\
& \Lambda:=\sup _{M \times[0, T]} \hat{A}_{f}^{+}=\max \left\{0, \sup _{M \times[0, T]}\left(A^{\prime}(u)-A(u) / u\right)\right\} \\
& \Sigma:=\sup _{M \times[0, T]} \hat{A}_{f f}^{+}=\max \left\{0, \sup _{M \times[0, T]}\left(u A^{\prime \prime}(u)-A^{\prime}(u)+A(u) / u\right)\right\} \\
& \kappa:=-\min \left\{0, \inf _{Q_{2 R, T}}\left(A^{\prime}(u)-A(u) / u\right), \inf _{Q_{2 R, T}} A^{\prime}(u)\right\} .
\end{aligned}
$$

Here, we denote by $v^{+}=\max \{0, v\}$ and $v^{-}=\min \{0, v\}$ the positive part and the negative part of a function $v$. Notice that if $M$ is compact, then $\lambda, \Lambda, \Sigma$ and $\kappa$ must be finite.

\section{SPACE-TIME GRADIENT ESTIMATES FOR POSITIVE SOLUTIONS}

Firstly, we have the following local space-time gradient estimate for (1.1)-(1.2).

Theorem 2.1. Let $(M, g(0))$ be a complete Riemannian manifold, and let $g(t)$ evolves by (1.21) for $t \in[0, T]$. Given $x_{0}$ and $R>0$, let $u$ be a positive solution to (1.1) in the cube $Q_{2 R, T}:=\{(x, t)$ : $\left.d\left(x, x_{0}, t\right) \leq 2 R, 0 \leq t \leq T\right\}$. Suppose that there exist constants $K_{1}, K_{2}, K_{3}, K_{4}, \gamma, \theta \geq 0$ such that

$$
\text { Ric } \geq-K_{1} g, \quad-K_{2} g \leq h \leq K_{3} g, \quad|\nabla h| \leq K_{4}
$$

and

$$
|\nabla q| \leq \gamma_{2 R}, \quad \Delta q \leq \theta_{2 R}
$$

on $Q_{2 R, T}$. Then for any $\alpha>1$ and $0<\varepsilon<1$, we have

$$
\begin{aligned}
& \frac{|\nabla u(x, t)|^{2}}{u^{2}(x, t)}-\alpha \frac{u_{t}(x, t)}{u(x, t)}-\alpha q(x, t)-\alpha \frac{A(u(x, t))}{u(x, t)} \\
& \leq \frac{n \alpha^{2}}{t}+\frac{C \alpha^{2}}{R^{2}}\left(\frac{\alpha^{2}}{\alpha-1}+\sqrt{K_{1}} R\right)+C \alpha^{2} K_{2}+n \alpha^{2} \lambda_{2 R} \\
& \quad+\left\{n \alpha ^ { 2 } \left[\alpha \theta_{2 R}+n \alpha^{2} \max \left\{K_{2}^{2}, K_{3}^{2}\right\}\right.\right. \\
& \quad+\frac{n \alpha^{2}}{4(1-\varepsilon)(\alpha-1)^{2}}\left((\alpha-1) \Lambda_{2 R}+\alpha \Sigma_{2 R}+2\left(K_{1}+(\alpha-1) K_{3}+K_{4}\right)\right)^{2} \\
& \left.\left.\quad+\frac{9}{8} n \alpha^{2} K_{4}+\frac{3}{4}\left(\frac{4 n \alpha^{2}}{\varepsilon}\right)^{\frac{1}{3}}(\alpha-1)^{\frac{2}{3}} \gamma_{2 R}^{\frac{4}{3}}\right]\right\}^{\frac{1}{2}}
\end{aligned}
$$

on $Q_{R, T}$, where $C$ is a constant that depends only on $n$.

Remark 2.2. We see that Theorem [2.1 covers [30, Theorem 1]. In fact, when $q(x, t)=A(u)=0$, from Theorem 2.1 we can get

$$
\begin{aligned}
\frac{|\nabla u(x, t)|^{2}}{u^{2}(x, t)}-\alpha \frac{u_{t}(x, t)}{u(x, t)} \leq & \frac{n \alpha^{2}}{t}+\frac{C \alpha^{2}}{R^{2}}\left(\frac{\alpha^{2}}{\alpha-1}+\sqrt{K_{1}} R\right)+C \alpha^{2} K_{2} \\
& +\left\{n \alpha ^ { 2 } \left[n \alpha^{2} \max \left\{K_{2}^{2}, K_{3}^{2}\right\}+\frac{9}{8} n \alpha^{2} K_{4}\right.\right. \\
& \left.\left.+\frac{n \alpha^{2}}{(1-\varepsilon)(\alpha-1)^{2}}\left(K_{1}+(\alpha-1) K_{3}+K_{4}\right)^{2}\right]\right\}^{\frac{1}{2}} .
\end{aligned}
$$


Let $\varepsilon \rightarrow 0+$, we thus get

$$
\begin{aligned}
\frac{|\nabla u(x, t)|^{2}}{u^{2}(x, t)}-\alpha \frac{u_{t}(x, t)}{u(x, t)} \leq & \frac{n \alpha^{2}}{t}+\frac{C \alpha^{2}}{R^{2}}\left(R \sqrt{K_{1}}+\frac{\alpha^{2}}{\alpha-1}\right)+C \alpha^{2} K_{2} \\
& +\frac{n \alpha^{2}}{\alpha-1}\left(K_{1}+(\alpha-1) K_{3}+K_{4}\right) \\
& +n \alpha^{2}\left(\max \left\{K_{2}, K_{3}\right\}+\sqrt{9 K_{4} / 8}\right)^{2} .
\end{aligned}
$$

To prove Theorem 2.1, we need the following two lemmas. Let $f=\log u$, by (1.1) we know that $f$ satisfies

$$
\Delta f=f_{t}+q+\frac{A(u)}{u}-|\nabla f|^{2}=f_{t}+q+\hat{A}(f)-|\nabla f|^{2}
$$

Set $F=t\left(|\nabla f|^{2}-\alpha f_{t}-\alpha q-\alpha \hat{A}\right)$. We have

Lemma 2.3 (Lemma 3 in [30]). Suppose the metric evolves by (1.2]). Then for any smooth function $f$, we have

and

$$
\frac{\partial}{\partial t}|\nabla f|^{2}=-2 h(\nabla f, \nabla f)+2\left\langle\nabla f, \nabla\left(f_{t}\right)\right\rangle
$$

$$
(\Delta f)_{t}=\Delta\left(f_{t}\right)-2\langle h, \operatorname{Hess} f\rangle-2\left\langle\operatorname{div} h-\frac{1}{2} \nabla\left(\operatorname{tr}_{g} h\right), \nabla f\right\rangle,
$$

where div $h$ is the divergence of $h$.

Lemma 2.4. Let $(M, g(t))$ satisfies the hypotheses of Theorem 2.1. Then for any $\delta \in\left(0, \frac{1}{\alpha}\right)$, we have

$$
\begin{aligned}
\left(\Delta-\partial_{t}\right) F \geq & \frac{2(1-\delta \alpha) t}{n}\left(|\nabla f|^{2}-f_{t}-q-\hat{A}\right)^{2}-\frac{F}{t}-2\langle\nabla f, \nabla F\rangle \\
& +\alpha t \hat{A}_{f}\left(|\nabla f|^{2}-f_{t}-q-\hat{A}\right)-2(\alpha-1) t\langle\nabla f, \nabla q\rangle \\
& -t\left(2(\alpha-1) \hat{A}_{f}+\alpha \hat{A}_{f f}+2 K_{1}+2(\alpha-1) K_{3}\right)|\nabla f|^{2} \\
& -3 \alpha t \sqrt{n} K_{4}|\nabla f|-\frac{\alpha t n}{2 \delta} \max \left\{K_{2}^{2}, K_{3}^{2}\right\}-\alpha t \Delta q .
\end{aligned}
$$

Proof. By the Bochner formula, (2.2) and Lemma 2.3, we calculate

$$
\begin{aligned}
\Delta F= & 2 t|\operatorname{Hess} f|^{2}+2 t \operatorname{Ric}(\nabla f, \nabla f)+2 t\langle\nabla f, \nabla \Delta f\rangle \\
& -\alpha t \Delta\left(f_{t}\right)-\alpha t \Delta q-\alpha t \hat{A}_{f} \Delta f-\alpha t \hat{A}_{f f}|\nabla f|^{2} \\
= & 2 t|\operatorname{Hess} f|^{2}+2 t \operatorname{Ric}(\nabla f, \nabla f)+2 t\langle\nabla f, \nabla \Delta f\rangle \\
& -\alpha t(\Delta f)_{t}-2 \alpha t\langle h, \operatorname{Hess} f\rangle-2 \alpha t\left\langle\operatorname{div} h-\frac{1}{2} \nabla\left(\operatorname{tr}_{g} h\right), \nabla f\right\rangle \\
& -\alpha t \Delta q-\alpha t a \hat{A}_{f} \Delta f-\alpha t a \hat{A}_{f f}|\nabla f|^{2} .
\end{aligned}
$$

By $(\underline{2.2})$ and the definition of $F$ we have

$$
\nabla \Delta f=-\frac{\nabla F}{t}-(\alpha-1)\left(\nabla\left(f_{t}\right)+\nabla q+\hat{A}_{f} \nabla f\right)
$$

and

$$
(\Delta f)_{t}=\frac{F}{t^{2}}-\frac{F_{t}}{t}-(\alpha-1)\left(f_{t t}+q_{t}+\hat{A}_{f} f_{t}\right)
$$

By Lemma 2.3 we also have

$$
\begin{aligned}
F_{t}= & |\nabla f|^{2}-\alpha f_{t}-\alpha q-\alpha \hat{A} \\
& +2 t\left\langle\nabla f,(\nabla f)_{t}\right\rangle-\alpha t\left(f_{t t}+q_{t}+\hat{A}_{f} f_{t}\right) \\
= & |\nabla f|^{2}-\alpha f_{t}-\alpha q-\alpha \hat{A} \\
& +2 t\left\langle\nabla f, \nabla\left(f_{t}\right)\right\rangle-2 \operatorname{th}(\nabla f, \nabla f)-\alpha t\left(f_{t t}+q_{t}+\hat{A}_{f} f_{t}\right) .
\end{aligned}
$$


It follows the above equalities that

$$
\begin{aligned}
\left(\Delta-\partial_{t}\right) F= & 2 t|\operatorname{Hess} f|^{2}+2 t \operatorname{Ric}(\nabla f, \nabla f)-\frac{F}{t}-2\langle\nabla f, \nabla F\rangle \\
& -2 \alpha t\langle h, \operatorname{Hess} f\rangle-2 \alpha t\left\langle\operatorname{div} h-\frac{1}{2} \nabla\left(\operatorname{tr}_{g} h\right), \nabla f\right\rangle \\
& +\alpha t \hat{A}_{f}\left(|\nabla f|^{2}-f_{t}-q-\hat{A}\right)-\alpha t \Delta q-2(\alpha-1) t\langle\nabla f, \nabla q\rangle \\
& -2(\alpha-1) \operatorname{th}(\nabla f, \nabla f)-2(\alpha-1) t \hat{A}_{f}|\nabla f|^{2}-\alpha t \hat{A}_{f f}|\nabla f|^{2}
\end{aligned}
$$

The assumption $-K_{2} g \leq h \leq K_{3} g$ implies

$$
|h|^{2} \leq n \max \left\{K_{2}^{2}, K_{3}^{2}\right\} .
$$

By Young's inequality,

$$
\begin{aligned}
\langle h, \operatorname{Hess} f\rangle & \leq \delta|\operatorname{Hess} f|^{2}+\frac{1}{4 \delta}|h|^{2} \\
& \leq \delta|\operatorname{Hess} f|^{2}+\frac{n}{4 \delta} \max \left\{K_{2}^{2}, K_{3}^{2}\right\}
\end{aligned}
$$

for any $\delta \in\left(0, \frac{1}{\alpha}\right)$. We also have

$$
\begin{aligned}
\left|\operatorname{div} h-\frac{1}{2} \nabla\left(\operatorname{tr}_{g} h\right)\right| & =\left|g^{i j} \nabla_{i} h_{j l}-\frac{1}{2} g^{i j} \nabla_{l} h_{i j}\right| \\
& \leq \frac{3}{2}|g||\nabla h| \leq \frac{3}{2} \sqrt{n} K_{4} .
\end{aligned}
$$

On the other hand,

$$
|\operatorname{Hess} f|^{2} \geq \frac{1}{n}(\Delta f)^{2}=\frac{1}{n}\left(|\nabla f|^{2}-f_{t}-q-\hat{A}\right)^{2} .
$$

Substituting (2.5), (2.6) and (2.7) into (2.4) and using the assumptions on bounds of Ric and $h$, we obtain the final inequality $(2 \cdot 3)$.

The proof of Theorem 2.1. By the assumption of bounds of Ricci tensor and the evolution of the metric, we know that $g(t)$ is uniformly equivalent to the initial metric $g(0)$ (see [ 8 , Corollary 6.11]), that is,

$$
e^{-2 K_{2} T} g(0) \leq g(t) \leq e^{2 K_{3} T} g(0)
$$

Then we know that $(M, g(t))$ is also complete for $t \in[0, T]$.

Let $\phi \in C^{\infty}((0,+\infty])$,

$$
\phi(s)= \begin{cases}1, & s \in[0,1], \\ 0, & s \in[2,+\infty)\end{cases}
$$

satisfies $\phi(s) \in[0,1], \phi^{\prime}(s) \leq 0, \phi^{\prime \prime}(s) \geq-C_{1}$ and $\frac{\left|\phi^{\prime}(s)\right|^{2}}{\phi(s)} \leq C_{1}$, where $C_{1}$ is an absolute constant. Define

$$
\eta(x, t)=\phi\left(\frac{r(x, t)}{R}\right),
$$

where $r(x, t)=d\left(x, x_{0}, t\right)$. Using the argument of [2], we can assume that the function $\eta(x, t)$ is $C^{2}$ with support in $Q_{2 R, T}$.

Define $G=\eta F$. For any $T_{1} \in(0, T]$, let $\left(x_{1}, t_{1}\right) \in Q_{2 R, T_{1}}$ at which $G$ attains its maximum, and without loss of generality, we can assume $G\left(x_{1}, t_{1}\right)>0$, and then $\eta\left(x_{1}, t_{1}\right)>0$ and $F\left(x_{1}, t_{1}\right)>0$. Hence, at $\left(x_{1}, t_{1}\right)$, we have

$$
\nabla G=0, \quad \Delta G \leq 0, \quad \partial_{t} G \geq 0 .
$$

Hence, we obtain

$$
\nabla F=-\frac{F}{\eta} \nabla \eta
$$


and

$$
\begin{aligned}
0 & \geq\left(\Delta-\partial_{t}\right) G \\
& =F\left(\Delta-\partial_{t}\right) \eta+\eta\left(\Delta-\partial_{t}\right) F+2\langle\nabla \eta, \nabla F\rangle .
\end{aligned}
$$

By the properties of $\phi$ and the Laplacian comparison theorem, we have

$$
\frac{|\nabla \eta|^{2}}{\eta} \leq \frac{C_{1}}{R^{2}}
$$

and

$$
\begin{aligned}
\Delta \eta & =\phi^{\prime} \frac{\Delta r}{R}+\phi^{\prime \prime} \frac{|\nabla r|^{2}}{R^{2}} \\
& \geq-\frac{\sqrt{C_{1}}}{R} \sqrt{(n-1) K_{1}} \operatorname{coth}\left(\sqrt{\frac{K_{1}}{n-1}} R\right)-\frac{C_{1}}{R^{2}} \\
& \geq-\frac{(n-1) \sqrt{C_{1}}}{R^{2}}-\frac{\sqrt{(n-1) C_{1} K_{1}}}{R}-\frac{C_{1}}{R^{2}} .
\end{aligned}
$$

By [30, p. 494], there exist a constant $C_{2}$ such that

$$
-F \eta_{t} \geq-C_{2} K_{2} F
$$

Substituting the above three inequalities into (2.9) and using (2.8), we obtain

$$
\begin{aligned}
0 & \geq-\left(\frac{(n-1) \sqrt{C_{1}}}{R^{2}}+\frac{\sqrt{(n-1) C_{1} K_{1}}}{R}+\frac{3 C_{1}}{R^{2}}+C_{2} K_{2}\right) F+\eta\left(\Delta-\partial_{t}\right) F \\
& =: \eta\left(\Delta-\partial_{t}\right) F-\left(\frac{C_{3}(n)}{R^{2}}+\frac{C_{4}(n)}{R} \sqrt{K_{1}}+C_{2} K_{2}\right) F .
\end{aligned}
$$

Let $\mu=\frac{\left|\nabla f\left(x_{1}, t_{1}\right)\right|^{2}}{F\left(x_{1}, t_{1}\right)}$, then, at $\left(x_{1}, t_{1}\right)$ we have

$$
\eta\langle\nabla f, \nabla F\rangle=-F\langle\nabla f, \nabla \eta\rangle \leq \frac{\sqrt{C_{1}}}{R} \eta^{\frac{1}{2}} F|\nabla f|
$$

and

$$
|\nabla f|^{2}-f_{t}-q-a \hat{A}=\left(\mu-\frac{t_{1} \mu-1}{t_{1} \alpha}\right) F .
$$

Therefore, at $\left(x_{1}, t_{1}\right)$, by Lemma 2.4 and (2.10), and using the inequality

$$
3 \alpha \sqrt{n} K_{4}|\nabla f| \leq 2 K_{4}|\nabla f|^{2}+\frac{9}{8} n \alpha^{2} K_{4},
$$

we obtain

$$
\begin{aligned}
0 \geq & \frac{2(1-\delta \alpha) t_{1}}{n} \eta\left(\mu-\frac{t_{1} \mu-1}{t_{1} \alpha}\right)^{2} F^{2}-\frac{\eta F}{t_{1}}-\frac{2 \sqrt{C_{1}}}{R} \eta^{\frac{1}{2}} \mu^{\frac{1}{2}} F^{\frac{3}{2}} \\
& +\alpha t_{1} \eta \hat{A}_{f}\left(\mu-\frac{t_{1} \mu-1}{t_{1} \alpha}\right) F-\alpha t_{1} \eta \Delta q-2(\alpha-1) t_{1} \eta\langle\nabla f, \nabla q\rangle \\
& -t_{1} \eta\left(2(\alpha-1) \hat{A}_{f}+\alpha \hat{A}_{f f}+2\left(K_{1}+(\alpha-1) K_{3}+K_{4}\right)\right) \mu F \\
& -\frac{9}{8} t_{1} \eta n \alpha^{2} K_{4}-\frac{t_{1} \eta n \alpha}{2 \delta} \max \left\{K_{2}^{2}, K_{3}^{2}\right\} \\
& -\left(\frac{C_{3}(n)}{R^{2}}+\frac{C_{4}(n)}{R} \sqrt{K_{1}}+C_{2} K_{2}\right) F
\end{aligned}
$$


Multiplying through by $t_{1} \eta$, we conclude that

$$
\begin{aligned}
0 \geq & \frac{2(1-\delta \alpha)}{n \alpha^{2}}\left(1+(\alpha-1) t_{1} \mu\right)^{2} G^{2}-\eta G-\frac{2 \sqrt{C_{1}}}{R} t_{1} \mu^{\frac{1}{2}} G^{\frac{3}{2}} \\
& +t_{1} \eta \hat{A}_{f}\left(1+(\alpha-1) t_{1} \mu\right) G-\alpha t_{1}^{2} \eta^{2} \Delta q-2(\alpha-1) t_{1}^{2} \eta^{2}\langle\nabla f, \nabla q\rangle \\
& -t_{1}^{2} \eta\left[2(\alpha-1) \hat{A}_{f}+\alpha \hat{A}_{f f}+2\left(K_{1}+(\alpha-1) K_{3}+K_{4}\right)\right] \mu G \\
& -\frac{9}{8} t_{1}^{2} \eta^{2} n \alpha^{2} K_{4}-\frac{t_{1}^{2} \eta^{2} n \alpha}{2 \delta} \max \left\{K_{2}^{2}, K_{3}^{2}\right\} \\
& -t_{1}\left(\frac{C_{3}(n)}{R^{2}}+\frac{C_{4}(n)}{R} \sqrt{K_{1}}+C_{2} K_{2}\right) G \\
= & \frac{2(1-\delta \alpha)}{n \alpha^{2}}\left(1+(\alpha-1) t_{1} \mu\right)^{2} G^{2}-\eta G-\frac{2 \sqrt{C_{1}}}{R} t_{1} \mu^{\frac{1}{2}} G^{\frac{3}{2}} \\
& +t_{1} \eta \hat{A}_{f} G-\alpha t_{1}^{2} \eta^{2} \Delta q-2(\alpha-1) t_{1}^{2} \eta^{2}\langle\nabla f, \nabla q\rangle \\
& -t_{1}^{2} \eta\left[(\alpha-1) \hat{A}_{f}+\alpha \hat{A}_{f f}+2\left(K_{1}+(\alpha-1) K_{3}+K_{4}\right)\right] \mu G \\
& -\frac{9}{8} t_{1}^{2} \eta^{2} n \alpha^{2} K_{4}-\frac{t_{1}^{2} \eta^{2} n \alpha}{2 \delta} \max \left\{K_{2}^{2}, K_{3}^{2}\right\} \\
& -t_{1}\left(\frac{C_{3}(n)}{R^{2}}+\frac{C_{4}(n)}{R} \sqrt{K_{1}}+C_{2} K_{2}\right) G
\end{aligned}
$$

Noticing that $0<\eta\left(x_{1}, t_{1}\right) \leq 1$, from the above inequalities we obtain

$$
\begin{aligned}
0 \geq & \frac{2(1-\delta \alpha)}{n \alpha^{2}} G^{2}+\frac{4(1-\delta \alpha)}{n \alpha^{2}}(\alpha-1) t_{1} \mu G^{2} \\
& +\frac{2(1-\delta \alpha)}{n \alpha^{2}}(\alpha-1)^{2} t_{1}^{2} \mu^{2} G^{2}-G-t_{1} \lambda_{2 R} G-\frac{2 \sqrt{C_{1}}}{R} t_{1} \mu^{\frac{1}{2}} G^{\frac{3}{2}} \\
& -t_{1}^{2}\left[(\alpha-1) \Lambda_{2 R}+\alpha \Sigma_{2 R}+2\left(K_{1}+(\alpha-1) K_{3}+K_{4}\right)\right] \mu G \\
& -2 t_{1}^{2}(\alpha-1) \gamma_{2 R} \mu^{\frac{1}{2}} G^{\frac{1}{2}}-\frac{9}{8} t_{1}^{2} n \alpha^{2} K_{4}-\frac{t_{1}^{2} n \alpha}{2 \delta} \max \left\{K_{2}^{2}, K_{3}^{2}\right\} \\
& -\alpha t_{1}^{2} \theta_{2 R}-t_{1}\left(\frac{C_{3}(n)}{R^{2}}+\frac{C_{4}(n)}{R} \sqrt{K_{1}}+C_{2} K_{2}\right) G .
\end{aligned}
$$

By Young's inequality, we have

$$
\begin{gathered}
\frac{2 \sqrt{C_{1}}}{R} \mu^{\frac{1}{2}} G^{\frac{3}{2}} \leq \frac{4(1-\delta \alpha)}{n \alpha^{2}}(\alpha-1) \mu G^{2}+\frac{n \alpha^{2} C_{1} G}{8(1-\delta \alpha)(\alpha-1) R^{2}}, \\
2(\alpha-1) \gamma_{2 R} \mu^{\frac{1}{2}} G^{\frac{1}{2}} \leq \frac{2(1-\delta \alpha) \varepsilon}{n \alpha^{2}}(\alpha-1)^{2} \mu^{2} G^{2}+\frac{3}{4}\left(\frac{2 n \alpha^{2}}{(1-\delta \alpha) \varepsilon}\right)^{\frac{1}{3}}(\alpha-1)^{\frac{2}{3}} \gamma_{2 R}^{\frac{4}{3}}
\end{gathered}
$$

and

$$
\begin{aligned}
& {\left[(\alpha-1) \Lambda_{2 R}+\alpha \Sigma_{2 R}+2\left(K_{1}+(\alpha-1) K_{3}+K_{4}\right)\right] \mu G } \\
\leq & \frac{2(1-\delta \alpha)(1-\varepsilon)}{n \alpha^{2}}(\alpha-1)^{2} \mu^{2} G^{2} \\
& +\frac{n \alpha^{2}}{8(1-\delta \alpha)(1-\varepsilon)(\alpha-1)^{2}}\left[(\alpha-1) \Lambda_{2 R}+\alpha \Sigma_{2 R}+2\left(K_{1}+(\alpha-1) K_{3}+K_{4}\right)\right]^{2},
\end{aligned}
$$


where $\varepsilon \in(0,1)$ is an arbitrary constant. Combining the above four inequalities, there exists a constant $C_{5}(n)$ that depends only on $n$, such that

$$
\begin{aligned}
0 \geq & \frac{2(1-\delta \alpha)}{n \alpha^{2}} G^{2} \\
& -\left[1+t_{1}\left(\lambda_{2 R}+\frac{C_{5}(n)}{R^{2}}\left(\frac{\alpha^{2}}{(1-\delta \alpha)(\alpha-1)}+\sqrt{K_{1}} R\right)+C_{2} K_{2}\right)\right] G \\
& -t_{1}^{2} \alpha \theta_{2 R}-t_{1}^{2} n \alpha^{2} \max \left\{K_{2}^{2}, K_{3}^{2}\right\} \\
& -t_{1}^{2} \frac{n \alpha^{2}}{8(1-\delta \alpha)(1-\varepsilon)(\alpha-1)^{2}} \\
& \cdot\left[(\alpha-1) \Lambda_{2 R}+\alpha \Sigma_{2 R}+2\left(K_{1}+(\alpha-1) K_{3}+K_{4}\right)\right]^{2} \\
& -\frac{9}{8} t_{1}^{2} n \alpha^{2} K_{4}-\frac{3}{4} t_{1}^{2}\left(\frac{2 n \alpha^{2}}{(1-\delta \alpha) \varepsilon}\right)^{\frac{1}{3}}(\alpha-1)^{\frac{2}{3}} \gamma_{2 R}^{\frac{4}{3}} .
\end{aligned}
$$

For a positive number $a$ and two nonnegative numbers $b, c$, from the inequality $a x^{2}-b x-c \leq 0$ we have $x \leq \frac{b}{a}+\sqrt{\frac{c}{a}}$. Hence, we obtain

$$
\begin{aligned}
G \leq & \frac{n \alpha^{2}}{2(1-\delta \alpha)}\left[1+t_{1}\left(\lambda_{2 R}+\frac{C_{5}(n)}{R^{2}}\left(\frac{\alpha^{2}}{(1-\delta \alpha)(\alpha-1)}+\sqrt{K_{1}} R\right)+C_{2} K_{2}\right)\right] \\
+ & t_{1}\left\{n \alpha ^ { 2 } \left[\alpha \theta_{2 R}+n \alpha^{2} \max \left\{K_{2}^{2}, K_{3}^{2}\right\}\right.\right. \\
+ & \frac{n \alpha^{2}}{8(1-\delta \alpha)(1-\varepsilon)(\alpha-1)^{2}} \\
\cdot & {\left[(\alpha-1) \Lambda_{2 R}+\alpha \Sigma_{2 R}+2\left(K_{1}+(\alpha-1) K_{3}+K_{4}\right)\right]^{2} } \\
+ & \left.\left.\frac{9}{8} n \alpha^{2} K_{4}+\frac{3}{4}\left(\frac{2 n \alpha^{2}}{(1-\delta \alpha) \varepsilon}\right)^{\frac{1}{3}}(\alpha-1)^{\frac{2}{3}} \gamma_{2 R}^{\frac{4}{3}}\right]\right\}^{\frac{1}{2}} .
\end{aligned}
$$

Now, by taking $\delta=\frac{1}{2 \alpha}$, and noticing that $d\left(x, x_{0}, T_{1}\right) \leq R$ implies $\eta\left(x, T_{1}\right)=1$, we can get

$$
\begin{aligned}
& \left(|\nabla f|^{2}-\alpha f_{1}-\alpha q_{t}-\alpha \hat{A}\right)\left(x, T_{1}\right)=\frac{F\left(x, T_{1}\right)}{T_{1}} \leq \frac{G\left(x_{1}, t_{1}\right)}{T_{1}} \\
& \leq \frac{n \alpha^{2}}{T_{1}}+\frac{C(n) \alpha^{2}}{R^{2}}\left(\frac{\alpha^{2}}{\alpha-1}+\sqrt{K_{1}} R\right)+C(n) \alpha^{2} K_{2}+n \alpha^{2} \lambda_{2 R} \\
& +\left\{n \alpha ^ { 2 } \left[\alpha \theta_{2 R}+n \alpha^{2} \max \left\{K_{2}^{2}, K_{3}^{2}\right\}\right.\right. \\
& \quad+\frac{n \alpha^{2}}{4(1-\varepsilon)(\alpha-1)^{2}}\left((\alpha-1) \Lambda_{2 R}+\alpha \Sigma_{2 R}+2\left(K_{1}+(\alpha-1) K_{3}+K_{4}\right)\right)^{2} \\
& \left.\left.\quad+\frac{9}{8} n \alpha^{2} K_{4}+\frac{3}{4}\left(\frac{4 n \alpha^{2}}{\varepsilon}\right)^{\frac{1}{3}}(\alpha-1)^{\frac{2}{3}} \gamma_{2 R}^{\frac{4}{3}}\right]\right\}^{\frac{1}{2}},
\end{aligned}
$$

where $C(n)$ is an appropriate constant that depends only $n$. Since $T_{1}$ is arbitrary, we complete the proof.

Remark 2.5. In the above proof, if we use $x \leq \frac{1}{2 a}\left(b+\sqrt{b^{2}+4 a c}\right)$ instead of $x \leq \frac{b}{a}+\sqrt{\frac{c}{a}}$ when we deal with $a x^{2}-b x-c \leq 0$, then a more appropriate $\delta$ may give a sharper estimate.

From the above local estimate, we get a global one: 
Corollary 2.6. Let $(M, g(0))$ be a complete noncompact Riemannian manifold without boundary, and let $g(t)$ evolves by (1.2) for $t \in[0, T]$. Suppose that there exist constant $K_{1}, K_{2}, K_{3}, K_{4}, \gamma, \theta \geq 0$ such that

and

$$
\text { Ric } \geq-K_{1} g, \quad-K_{2} g \leq h \leq K_{3} g, \quad|\nabla h| \leq K_{4}
$$

$$
|\nabla q| \leq \gamma, \quad \Delta q \leq \theta
$$

If $u$ is a positive solution to (1.1), then for any $\alpha>1$, we have

$$
\begin{aligned}
& \frac{|\nabla u(x, t)|^{2}}{u^{2}(x, t)}-\alpha \frac{u_{t}(x, t)}{u(x, t)}-\alpha q(x, t)-\alpha \frac{A(u(x, t))}{u(x, t)} \\
\leq & \frac{n \alpha^{2}}{t}+C^{\prime}\left(K_{1}+K_{2}+K_{3}+K_{4}+\sqrt{K_{4}}+\sqrt{\theta}+\gamma^{\frac{2}{3}}+\lambda+\Lambda+\Sigma\right)
\end{aligned}
$$

on $M \times[0, T]$, where $C^{\prime}$ is a constant that depends only on $n, \alpha$.

Proof. By the uniform equivalence of $g(t)$, we know that $(M, g(t))$ is complete noncompact for $t \in[0, T]$. Now we choose $\varepsilon=\varepsilon_{0}$ in (2.1), where $\varepsilon_{0}$ is an arbitrary fixed number in $(0,1)$. Let $R \rightarrow+\infty$ in (2.1), and using the inequality $\sqrt{x+y} \leq \sqrt{x}+\sqrt{y}$ holds for any $x, y \geq 0$, we complete the proof.

We now consider the case that the manifold $M$ is closed. By Lemma 2.4, we have a global gradient estimate on a closed Riemannian manifold.

Theorem 2.7. Let $(M, g(t))$ be a closed Riemannian manifold, where $g(t)$ evolves by (1.2) for $t \in[0, T]$ and satisfies

$$
\text { Ric } \geq-K_{1} g, \quad-K_{2} g \leq h \leq K_{3} g, \quad|\nabla h| \leq K_{4} .
$$

If $u$ is a positive solution to (1.1), and $q(x, t)$ satisfies

$$
|\nabla q| \leq \gamma, \quad \Delta q \leq \theta .
$$

Then for any $\alpha>1$, we have

$$
\begin{aligned}
& \frac{|\nabla u(x, t)|^{2}}{u^{2}(x, t)}-\alpha \frac{u_{t}(x, t)}{u(x, t)}-\alpha q(x, t)-\alpha \frac{A(u(x, t))}{u(x, t)} \\
\leq & \frac{n \alpha^{2}}{2 t}+\frac{n \alpha^{2}}{\alpha-1}\left(K_{1}+(\alpha-1) K_{3}+K_{4}\right)+n \alpha^{2}\left(\max \left\{K_{2}, K_{3}\right\}+\frac{3}{4} \sqrt{2 K_{4}}\right) \\
& +\alpha^{\frac{3}{2}} \sqrt{n \theta}+\left(\frac{n \alpha^{2}}{2}(\alpha-1)^{\frac{1}{2}}+\sqrt{n} \alpha(\alpha-1)^{\frac{1}{4}}\right) \gamma^{\frac{2}{3}}+\frac{n \alpha^{2}}{2}(\lambda+\Lambda)+\frac{n \alpha^{3}}{2(\alpha-1)} \Sigma
\end{aligned}
$$

on $M \times(0, T]$.

Proof. We use the same symbols $f, F$ as above. Set

$$
\begin{aligned}
\bar{F}(x, t)= & F(x, t)-\frac{n \alpha^{2}}{\alpha-1}\left(K_{1}+(\alpha-1) K_{3}+K_{4}\right) t \\
& -n \alpha^{2}\left(\max \left\{K_{2}, K_{3}\right\}+\frac{3}{4} \sqrt{2 K_{4}}\right) t-\alpha^{\frac{3}{2}} \sqrt{n \theta} t \\
& -\left(\frac{n \alpha^{2}}{2}(\alpha-1)^{\frac{1}{2}}+\sqrt{n} \alpha(\alpha-1)^{\frac{1}{4}}\right) \gamma^{\frac{2}{3}} t-\frac{n \alpha^{2}}{2}(\lambda+\Lambda) t-\frac{n \alpha^{3}}{2(\alpha-1)} \Sigma t .
\end{aligned}
$$

If $\bar{F}(x, t) \leq \frac{n \alpha^{2}}{2}$ for any $(x, t) \in M \times(0, T]$, the proof is complete.

If (2.13) doesn't hold, then at the maximal point $\left(x_{0}, t_{0}\right)$ of $\bar{F}(x, t)$, we have

$$
\bar{F}\left(x_{0}, t_{0}\right)>\frac{n \alpha^{2}}{2} .
$$

As $\bar{F}(x, 0)=0$, we know that $t_{0}>0$ here. Then applying the maximum principle, we have

$$
\nabla \bar{F}\left(x_{0}, t_{0}\right)=0, \quad \Delta \bar{F}\left(x_{0}, t_{0}\right)=0, \quad \partial_{t} \bar{F}\left(x_{0}, t_{0}\right)=0 .
$$


Therefore, we obtain

$$
0 \geq\left(\Delta-\partial_{t}\right) \bar{F} \geq\left(\Delta-\partial_{t}\right) F .
$$

Using Lemma 2.4 inequality (2.11) and the fact that

$$
\begin{aligned}
|\nabla f|^{2}-f_{t}-q-\hat{A} & =\frac{1}{\alpha}\left(|\nabla f|^{2}-\alpha f_{t}-\alpha q-\alpha \hat{A}\right)+\frac{\alpha-1}{\alpha}|\nabla f|^{2} \\
& =\frac{1}{\alpha} \frac{F}{t_{0}}+\frac{\alpha-1}{\alpha}|\nabla f|^{2},
\end{aligned}
$$

we obtain

$$
\begin{aligned}
0 \geq & \frac{2(1-\delta \alpha) t_{0}}{n \alpha^{2}}\left(\frac{F}{t_{0}}\right)^{2}+\frac{4(1-\delta \alpha)(\alpha-1) t_{0}}{n \alpha^{2}}|\nabla f|^{2} \frac{F}{t_{0}} \\
& +\frac{2(1-\delta \alpha)(\alpha-1)^{2} t_{0}}{n \alpha^{2}}|\nabla f|^{4}-\frac{F}{t_{0}}-t_{0} \lambda \frac{F}{t_{0}} \\
& -2 t_{0}(\alpha-1) \gamma|\nabla f|-\frac{9}{8} t_{0} n \alpha^{2} K_{4}-t_{0} \alpha \theta-\frac{t_{0} n \alpha}{2 \delta} \max \left\{K_{2}^{2}, K_{3}^{2}\right\} \\
& -t_{0}\left((\alpha-1) \Lambda+\alpha \Sigma+2\left(K_{1}+(\alpha-1) K_{3}+K_{4}\right)\right)|\nabla f|^{2} .
\end{aligned}
$$

By

$$
\begin{aligned}
& \frac{F}{t_{0}}= \frac{\bar{F}}{t_{0}}+\frac{n \alpha^{2}}{\alpha-1}\left(K_{1}+(\alpha-1) K_{3}+K_{4}\right)+\frac{3}{2} n \alpha^{2} \sqrt{K_{4}} \\
&+\left(\frac{n \alpha^{2}}{2}(\alpha-1)^{\frac{1}{2}}+\sqrt{2 n} \alpha(\alpha-1)^{\frac{1}{4}}\right) \gamma^{\frac{2}{3}} \\
&+n \alpha^{2} A_{1}+\frac{n \alpha^{3}}{2(\alpha-1)} A_{2}>0, \\
& 2 t_{0}(\alpha-1) \gamma|\nabla f|^{2} \leq t_{0}(\alpha-1)^{\frac{3}{2}} \gamma^{\frac{2}{3}}|\nabla f|^{2}+t_{0}(\alpha-1)^{\frac{1}{2}} \gamma^{\frac{4}{3}},
\end{aligned}
$$

and using the inequality $a x^{2}-b x \geq-\frac{b^{2}}{4 a}$ holds for $a>0, b \geq 0$, we obtain

$$
\begin{aligned}
0 \geq & \frac{2(1-\delta \alpha) t_{0}}{n \alpha^{2}}\left(\frac{F}{t_{0}}\right)^{2}-\frac{F}{t_{0}}-t_{0} \lambda \frac{F}{t_{0}}-\frac{9}{8} t_{0} n \alpha^{2} K_{4}-t_{0} \alpha \theta \\
& -\frac{t_{0} n \alpha}{2 \delta} \max \left\{K_{2}^{2}, K_{3}^{2}\right\}-t_{0}(\alpha-1)^{\frac{1}{2}} \gamma^{\frac{4}{3}}-\frac{t_{0} n \alpha^{2}}{8(1-\delta \alpha)(\alpha-1)^{2}} E^{2},
\end{aligned}
$$

where

$$
E=(\alpha-1)^{\frac{3}{2}} \gamma^{\frac{2}{3}}+(\alpha-1) \Lambda+\alpha \Sigma+2\left(K_{1}+(\alpha-1) K_{3}+K_{4}\right) .
$$

For a positive number $a$ and two nonnegative numbers $b, c$, from the inequality $a x^{2}-b x-c \leq 0$ we have

$$
x \leq \frac{1}{2 a}\left(b+\sqrt{b^{2}+4 a c}\right) .
$$

Hence, we obtain

$$
\begin{aligned}
\frac{F}{t_{0}} \leq & \frac{n \alpha^{2}}{4(1-\delta \alpha) t_{0}}\left\{1+t_{0} \lambda+\left[\left(1+t_{0} \lambda\right)^{2}\right.\right. \\
& +\frac{8(1-\delta \alpha) t_{0}}{n \alpha^{2}}\left(\frac{n \alpha^{2} t_{0}}{8(1-\delta \alpha)(\alpha-1)^{2}} E^{2}\right. \\
& \left.\left.\left.+t_{0}(\alpha-1)^{\frac{1}{2}} \gamma^{\frac{4}{3}}+\frac{9}{8} t_{0} n \alpha^{2} K_{4}+t_{0} \alpha \theta+\frac{t_{0} n \alpha}{2 \delta} \max \left\{K_{2}^{2}, K_{3}^{2}\right\}\right)\right]^{\frac{1}{2}}\right\} .
\end{aligned}
$$


Using the inequality $\sqrt{x+y} \leq \sqrt{x}+\sqrt{y}$ holds for any $x, y \geq 0$, we obtain

$$
\begin{aligned}
\frac{F}{t_{0}} \leq & \frac{n \alpha^{2}}{4(1-\delta \alpha) t_{0}}\left\{1+t_{0} \lambda+\left[\left(1+t_{0} \lambda\right)^{2}+\frac{4(1-\delta \alpha) t_{0}^{2}}{\delta \alpha} \max \left\{K_{2}^{2}, K_{3}^{2}\right\}\right]^{\frac{1}{2}}\right\} \\
& +\frac{n \alpha^{2}}{4(1-\delta \alpha) t_{0}}\left\{\frac { 8 ( 1 - \delta \alpha ) t _ { 0 } ^ { 2 } } { n \alpha ^ { 2 } } \left[\frac{n \alpha^{2}}{8(1-\delta \alpha)(\alpha-1)^{2}} E^{2}\right.\right. \\
& \left.\left.+(\alpha-1)^{\frac{1}{2}} \gamma^{\frac{4}{3}}+\frac{9}{8} n \alpha^{2} K_{4}+\alpha \theta\right]\right\}^{\frac{1}{2}} .
\end{aligned}
$$

Now, by taking $\delta=\frac{t_{0} \max \left\{K_{2}, K_{3}\right\}}{1+t_{0} \lambda+2 t_{0} \max \left\{K_{2}, K_{3}\right\}} \cdot \frac{1}{\alpha} \in\left(0, \frac{1}{\alpha}\right)$ for $\max \left\{K_{2}, K_{3}\right\} \neq 0$. However, from Lemma 2.4 we know that we can choose $\delta=0$ if $K_{2}=K_{3}=0$. Therefore, in any case, we can get

$$
\begin{aligned}
& \frac{n \alpha^{2}}{4(1-\delta \alpha) t_{0}}\left\{1+t_{0} \lambda+\left[\left(1+t_{0} \lambda\right)^{2}+\frac{4(1-\delta \alpha) t_{0}^{2}}{\delta \alpha} \max \left\{K_{2}^{2}, K_{3}^{2}\right\}\right]^{\frac{1}{2}}\right\} \\
= & \frac{n \alpha^{2}}{2 t_{0}}+\frac{n \alpha^{2}}{2} \lambda+n \alpha^{2} \max \left\{K_{2}, K_{3}\right\}
\end{aligned}
$$

and

$$
\frac{n \alpha^{2}}{4(1-\delta \alpha) t_{0}}\left(\frac{8(1-\delta \alpha) t_{0}^{2}}{n \alpha^{2}}\right)^{\frac{1}{2}}=\left(\frac{1+t_{0} \lambda+2 t_{0} \max \left\{K_{2}, K_{3}\right\}}{2\left(1+t_{0} \lambda+t_{0} \max \left\{K_{2}, K_{3}\right\}\right)}\right)^{\frac{1}{2}} \sqrt{n} \alpha \leq \sqrt{n} \alpha .
$$

Therefore, again according to $\sqrt{x+y} \leq \sqrt{x}+\sqrt{y}$, we obtain

$$
\begin{aligned}
\frac{F}{t_{0}} \leq & \frac{n \alpha^{2}}{2 t_{0}}+\frac{n \alpha^{2}}{2} \lambda+n \alpha^{2} \max \left\{K_{2}, K_{3}\right\} \\
& +\sqrt{n} \alpha\left\{\frac{\sqrt{n} \alpha}{2 \sqrt{2}(\alpha-1)} \sqrt{\frac{1+t_{0} \lambda+2 t_{0} \max \left\{K_{2}, K_{3}\right\}}{1+t_{0} \lambda+t_{0} \max \left\{K_{2}, K_{3}\right\}}} E\right. \\
& \left.+(\alpha-1)^{\frac{1}{4}} \gamma^{\frac{2}{3}}+\frac{3}{2 \sqrt{2}} \sqrt{n} \alpha \sqrt{K_{4}}+\sqrt{\alpha \theta}\right\} \\
\leq & \frac{n \alpha^{2}}{2 t_{0}}+\frac{n \alpha^{2}}{2} \lambda+n \alpha^{2} \max \left\{K_{2}, K_{3}\right\} \\
& +\frac{n \alpha^{2}}{2(\alpha-1)} E+\sqrt{n} \alpha(\alpha-1)^{\frac{1}{4}} \gamma^{\frac{2}{3}}+\frac{3}{4} n \alpha^{2} \sqrt{2 K_{4}}+\alpha^{\frac{3}{2}} \sqrt{n \theta} .
\end{aligned}
$$

Substituting $E$ into the above inequality yields

$$
\begin{aligned}
\frac{F}{t_{0}} \leq & \frac{n \alpha^{2}}{2 t_{0}}+\frac{n \alpha^{2}}{\alpha-1}\left(K_{1}+(\alpha-1) K_{3}+K_{4}\right) \\
& +n \alpha^{2}\left(\max \left\{K_{2}, K_{3}\right\}+\frac{3}{4} \sqrt{2 K_{4}}\right)+\alpha^{\frac{3}{2}} \sqrt{n \theta} \\
& +\left(\frac{n \alpha^{2}}{2}(\alpha-1)^{\frac{1}{2}}+\sqrt{n} \alpha(\alpha-1)^{\frac{1}{4}}\right) \gamma^{\frac{2}{3}}+\frac{n \alpha^{2}}{2}(\lambda+\Lambda)+\frac{n \alpha^{3}}{2(\alpha-1)} \Sigma .
\end{aligned}
$$

This implies that $\bar{F}\left(x_{0}, t_{0}\right) \leq \frac{n \alpha^{2}}{2}$, in contradiction with our assumption. So (2.13) holds.

Remark 2.8. In [30, Theorem 6], The coefficient of $\frac{1}{t}$ in the right hand side of the gradient inequality is $n \alpha^{2}$. We see Theorem 2.7 extends and improves Sun's estimate.

Remark 2.9. In Theorem 2.1 if $K_{1}=K_{4}=\Sigma_{2 R}=0$, we can let $\alpha \rightarrow 1$. Similarly, in Corollary 2.6 and Theorem 2.7, if $K_{1}=K_{4}=\Sigma=0$, we can also let $\alpha \rightarrow 1$. 
Similar to [30, Corollary 8], integrating the gradient estimate in space-time as in [18] or [10, we can derive the following parabolic Harnack type inequality.

Corollary 2.10. Let $(M, g(0))$ be a complete noncompact Riemannian manifold without boundary or a closed Riemannian manifold. Assume that $g(t)$ evolves by (1.2) for $t \in[0, T]$ and satisfies

$$
\text { Ric } \geq-K_{1} g, \quad-K_{2} g \leq h \leq K_{3} g, \quad|\nabla h| \leq K_{4} .
$$

If $u$ is a positive solution to (1.1), and $q(x, t)$ satisfies

$$
|\nabla q| \leq \gamma, \quad \Delta q \leq \theta .
$$

Then for any $\left(x_{1}, t_{1}\right),\left(x_{2}, t_{2}\right)$ in $M \times(0, T]$ such that $t_{1}<t_{2}$, we have

$$
u\left(x_{1}, t_{1}\right) \leq u\left(x_{2}, t_{2}\right)\left(\frac{t_{2}}{t_{1}}\right)^{\tau n \alpha} \exp \left(\frac{\alpha Z}{4\left(t_{2}-t_{1}\right)}+C \frac{t_{2}-t_{1}}{\alpha} K\right),
$$

for any $\alpha>1$, where

$$
\begin{gathered}
\tau= \begin{cases}1, & \text { if }(M, g(0)) \text { is complete noncompact without boundary, } \\
\frac{1}{2}, & \text { if }(M, g(0)) \text { is closed }\end{cases} \\
K=K_{1}+K_{2}+K_{3}+K_{4}+\sqrt{K_{4}}+\gamma+\sqrt{\theta}+\gamma^{\frac{2}{3}}+\lambda+\Lambda+\Sigma,
\end{gathered}
$$

$C$ is a constant that depends only on $n, \alpha$, and

$$
Z=\inf _{\zeta} \int_{0}^{1}\left|\zeta^{\prime}(s)\right|_{\sigma(s)}^{2} d s
$$

is the infimum over smooth curves $\zeta$ jointing $x_{2}$ and $x_{1}\left(\zeta(0)=x_{2}, \zeta(1)=x_{1}\right)$ of the averaged square velocity of $\zeta$ measured at time $\sigma(s)=(1-s) t_{2}+s t_{1}$.

Proof. The gradient estimate in Corollary 2.6 and Theorem 2.7 can both be written as

$$
\frac{|\nabla u(x, t)|^{2}}{u^{2}(x, t)}-\alpha \frac{u_{t}(x, t)}{u(x, t)} \leq \frac{\tau n \alpha^{2}}{t}+C(n, \alpha) K,
$$

for any $\alpha>1$. Take any curve $\zeta$ satisfying the assumption and define

$$
l(s)=\log u(\zeta(s), \sigma(s)) .
$$

Then $l(0)=\log u\left(x_{2}, t_{2}\right)$ and $l(1)=\log u\left(x_{1}, t_{1}\right)$. A direct computation yields

$$
\begin{aligned}
\frac{d l(s)}{d s} & =\left(t_{2}-t_{1}\right)\left(\frac{\nabla u}{u} \frac{\zeta^{\prime}(s)}{t_{2}-t_{1}}-\frac{u_{t}}{u}\right) \\
& \leq \frac{\alpha\left|\zeta^{\prime}(s)\right|_{\sigma}^{2}}{4\left(t_{2}-t_{1}\right)}+\frac{t_{2}-t_{1}}{\alpha}\left(\frac{\tau n \alpha^{2}}{\sigma(s)}+C K\right) .
\end{aligned}
$$

Integrating this inequality over $\zeta(s)$, we have

$$
\begin{aligned}
\log \frac{u\left(x_{1}, t_{1}\right)}{u\left(x_{2}, t_{2}\right)} & =\int_{0}^{1} \frac{d l(s)}{d s} d s \\
& \leq \int_{0}^{1} \frac{\alpha\left|\zeta^{\prime}(s)\right|_{\sigma}^{2}}{4\left(t_{2}-t_{1}\right)}+C \frac{t_{2}-t_{1}}{\alpha} K+\tau n \alpha \log \frac{t_{2}}{t_{1}},
\end{aligned}
$$

which implies the corollary. 


\section{Elliptic TyPe GRAdient ESTimates FOR BOUNDED POSITIVE SOLUtions}

Now we establish elliptic type gradient estimates for (1.1) $-(1.2)$. Firstly we give the local version.

Theorem 3.1. Let $(M, g(t))$ be a complete solution to (1.2) for $t \in[0, T]$ and let $u$ be a positive solution to (1.1). Suppose that there exist constants $L>0$ and $K \geq 0$, such that $u \leq L$ and

$$
\text { Ric } \geq-K_{1} g, \quad h \geq-K_{2} g
$$

on $Q_{2 R, T}$. Then we have

$$
\frac{|\nabla u(x, t)|}{u(x, t)} \leq \tilde{C}\left(\frac{1}{\sqrt{t}}+\frac{1}{R}+\sqrt{H}\right)\left(1+\log \frac{L}{u(x, t)}\right)
$$

on $Q_{R, T}$, where $\tilde{C}$ is a constant that depends only on $n$ and

$$
H=K_{1}+K_{2}+\max _{Q_{2 R, T}}(|q|-q)+\max _{Q_{2 R, T}} \frac{|\nabla q|}{\sqrt{|q|}}+\kappa_{2 R} .
$$

Remark 3.2. In [6, Theorem 1.9], the authors gave a elliptic type gradient estimate for bounded positive solutions of (1.1) with a convection term on a complete manifold, where the metric does not depend on time. In the estimate of [6, Theorem 1.9], the upper bound induced by the term $A(u)$ is

$$
-\min \left\{0, \min _{Q_{2 R, T}}\left(A^{\prime}(u)-A(u) / u\right)\right\}-\min \left\{0, \min _{Q_{2 R, T}}(A(u) / u)\right\}
$$

instead of $\kappa_{2 R}$ here. Compare with [6. Theorem 1.9], we see that our estimate (3.3) is sharper. In fact, in general, for real numbers $x, y$, a direct calculation yields

$$
\min \{0, x\}+\min \{0, y\} \leq \min \{0, x+y\} .
$$

Similarly, for two functions $f, g$ on the same domain $D$, the following obvious fact holds:

$$
\min _{D} f+\min _{D} g \leq \min _{D}(f+g) .
$$

By the above two inequalities we obtain

$$
\begin{aligned}
& \min \left\{0, \min _{Q_{2 R, T}}\left(A^{\prime}(u)-A(u) / u\right)\right\}+\min \left\{0, \min _{Q_{2 R, T}}(A(u) / u)\right\} \\
\leq & \min \left\{0, \min _{Q_{2 R, T}}\left(A^{\prime}(u)-A(u) / u\right)+\min _{Q_{2 R, T}}(A(u) / u)\right\} \\
\leq & \min \left\{0, \min _{Q_{2 R, T}} A^{\prime}(u)\right\} .
\end{aligned}
$$

On the other hand, it is obvious that

$$
\begin{aligned}
& \min \left\{0, \min _{Q_{2 R, T}}\left(A^{\prime}(u)-A(u) / u\right)\right\}+\min \left\{0, \min _{Q_{2 R, T}}(A(u) / u)\right\} \\
\leq & \min \left\{0, \min _{Q_{2 R, T}}\left(A^{\prime}(u)-A(u) / u\right)\right\} .
\end{aligned}
$$

In conclusion,

$$
\begin{aligned}
& \min \left\{0, \min _{Q_{2 R, T}}\left(A^{\prime}(u)-A(u) / u\right)\right\}+\min \left\{0, \min _{Q_{2 R, T}}(A(u) / u)\right\} \\
\leq & \min \left\{0, \min _{Q_{2 R, T}}\left(A^{\prime}(u)-A(u) / u\right), \min _{Q_{2 R, T}} A^{\prime}(u)\right\}=-\kappa_{2 R} .
\end{aligned}
$$

That is,

$$
\kappa_{2 R} \leq-\min \left\{0, \min _{Q_{2 R, T}}\left(A^{\prime}(u)-A(u) / u\right)\right\}-\min \left\{0, \min _{Q_{2 R, T}}(A(u) / u)\right\}
$$


And we will see in the proof of Theorem 3.1 that this sharper estimate comes from a more careful treatment of the term $\hat{A}_{f}+\frac{\hat{A}(f)}{1-f}$. However, the treatment we give here is not necessarily optimal. It is possible that a sharper estimate will be applied to more equations.

Now we are ready to prove Theorem 3.1. Noticing that if $0<u \leq L$ is a solution to (1.1), then $\tilde{u}=\frac{u}{L}$ is a solution to the equation

$$
\left(\Delta-q(x, t)-\partial_{t}\right) u(x, t)=\frac{1}{L} A(L \tilde{u}(x, t))
$$

and $0<\tilde{u} \leq 1$. Hence, we can assume that $0<u \leq 1$ in the proof of Theorem 3.1. Similar to the proof of Theorem 2.1, we need a auxiliary lemma. We still set $f=\log u \leq 0$ and $\hat{A}(f)=\frac{A(u)}{u}$. In this case, we define $w=|\nabla \log (1-f)|^{2}$ and $F(x, t)=t w(x, t)$.

Lemma 3.3. Let $(M, g(t))$ be a complete solution to (1.2) for $t \in[0, T]$ and let $u \in(0,1]$ be a solution to (1.1). Suppose that there exists a constant $K \geq 0$, such that

$$
\text { Ric }+h \geq-K g
$$

on $Q_{2 R, T}$. Then we have

$$
\begin{aligned}
\left(\Delta-\partial_{t}\right) F \geq & \frac{2(1-f)}{t} F^{2}-2\left(K-\hat{A}_{f}-\frac{q}{1-f}-\frac{\hat{A}(f)}{1-f}\right) F \\
& -\frac{F}{t}-2 f\langle\nabla \log (1-f), \nabla F\rangle-\frac{2 t}{1-f}\langle\nabla \log (1-f), \nabla q\rangle .
\end{aligned}
$$

on $Q_{2 R, T}$.

Proof. By the Bochner formula we have

$$
\begin{aligned}
\Delta F= & 2 t|\operatorname{Hess} \log (1-f)|^{2}+2 t \operatorname{Ric}(\nabla \log (1-f), \nabla \log (1-f)) \\
& +2 t\langle\nabla \log (1-f), \nabla \Delta \log (1-f)\rangle .
\end{aligned}
$$

However, by (2.21),

$$
\Delta \log (1-f)=-\frac{\Delta f}{1-f}-\frac{|\nabla f|^{2}}{(1-f)^{2}}=-f w-\frac{f_{t}+q+\hat{A}}{1-f} .
$$

Therefore, we obtain

$$
\begin{aligned}
\Delta F= & 2 t|\operatorname{Hess} \log (1-f)|^{2}+2 t \operatorname{Ric}(\nabla \log (1-f), \nabla \log (1-f)) \\
& +\frac{2(1-f)}{t} F^{2}-2 f\langle\nabla \log (1-f), \nabla F\rangle \\
& +\frac{2}{1-f}\left(f_{t}+q+\hat{A}\right) F-\frac{2 t}{1-f}\left\langle\nabla \log (1-f), \nabla\left(f_{t}\right)\right\rangle \\
& -\frac{2 t}{1-f}\langle\nabla \log (1-f), \nabla q\rangle-\frac{2 t \hat{A}_{f}}{1-f}\langle\nabla \log (1-f), \nabla f\rangle .
\end{aligned}
$$

On the other hand, by the first equality of Lemma 2.3.

$$
\begin{aligned}
F_{t}= & -2 \operatorname{th}(\nabla \log (1-f), \nabla \log (1-f)) \\
& +2 t\left\langle\nabla \log (1-f), \nabla\left((\log (1-f))_{t}\right)\right\rangle+\frac{F}{t} \\
= & -2 t h(\nabla \log (1-f), \nabla \log (1-f)) \\
& -\frac{2 t}{1-f}\left\langle\nabla \log (1-f), \nabla\left(f_{t}\right)\right\rangle+\frac{2 f_{t} F}{1-f}+\frac{F}{t} .
\end{aligned}
$$


Combining the above two equalities, we get

$$
\begin{aligned}
\left(\Delta-\partial_{t}\right) F \geq & \frac{2(1-f)}{t} F^{2}+2 t(\operatorname{Ric}+h)(\nabla \log (1-f), \nabla \log (1-f)) \\
& +2 \hat{A}_{f} F+\frac{2}{1-f}(q+\hat{A}) F-2 f\langle\nabla \log (1-f), \nabla F\rangle \\
& -\frac{2 t}{1-f}\langle\nabla \log (1-f), \nabla q\rangle-\frac{F}{t} .
\end{aligned}
$$

The lemma follows from the assumption on bound of Ric $+h$.

Remark 3.4. It is easy to see that we don't need any assumption on the Ricci tensor if geometric flow (1.2) is the Ricci flow, i.e., $h=-$ Ric.

The proof of Theorem 3.1. Choosing $\phi$ and $\eta$ as in the proof of Theorem 2.1. For any $T_{1} \in(0, T]$, let $\left(x_{1}, t_{1}\right) \in Q_{2 R, T_{1}}$, at which $G(x, t)=\eta(x, t) F(x, t)$ attains its maximum, and without loss of generality, we can assume $G\left(x_{1}, t_{1}\right)>0$, and then $\eta\left(x_{1}, t_{1}\right)>0$ and $F\left(x_{1}, t_{1}\right)>0$. By Lemma 3.3 and a similar argument as in the proof of Theorem 2.1 we have at $\left(x_{1}, t_{1}\right)$,

$$
\begin{aligned}
0 \geq & \frac{2 \eta(1-f)}{t_{1}} F^{2}-2 \eta\left(K_{1}+K_{2}-\hat{A}_{f}-\frac{q}{1-f}-\frac{\hat{A}(f)}{1-f}\right) F \\
& -2 \eta f\langle\nabla \log (1-f), \nabla F\rangle-\frac{2 t_{1} \eta}{1-f}\langle\nabla \log (1-f), \nabla q\rangle \\
& -\frac{\eta F}{t_{1}}-\left(\frac{C_{3}(n)}{R^{2}}+\frac{C_{4}(n)}{R} \sqrt{K_{1}}+C_{2} K_{2}\right) F \\
= & \frac{2 \eta(1-f)}{t_{1}} F^{2}-2 \eta\left(K_{1}+K_{2}-\hat{A}_{f}-\frac{q}{1-f}-\frac{\hat{A}(f)}{1-f}\right) F \\
& +2 f\langle\nabla \log (1-f), \nabla \eta\rangle F-\frac{2 t_{1} \eta}{1-f}\langle\nabla \log (1-f), \nabla q\rangle \\
& -\frac{\eta F}{t_{1}}-\left(\frac{C_{3}(n)}{R^{2}}+\frac{C_{4}(n)}{R} \sqrt{K_{1}}+C_{2} K_{2}\right) F .
\end{aligned}
$$

Multiplying both sides of the above inequality by $t_{1} \eta$, we have

$$
\begin{aligned}
0 \geq & 2(1-f) G^{2}-2 t_{1} \eta\left(K_{1}+K_{2}-\hat{A}_{f}-\frac{q}{1-f}-\frac{\hat{A}(f)}{1-f}\right) G \\
& +2 t_{1} f\langle\nabla \log (1-f), \nabla \eta\rangle G-\frac{2 t_{1}^{2} \eta^{2}}{1-f}\langle\nabla \log (1-f), \nabla q\rangle \\
& -\eta G-t_{1}\left(\frac{C_{3}(n)}{R^{2}}+\frac{C_{4}(n)}{R} \sqrt{K_{1}}+C_{2} K_{2}\right) G .
\end{aligned}
$$

Noticing that $f \leq 0$, by Young's inequality,

$$
\begin{aligned}
-2 t_{1} f\langle\nabla \log (1-f), \nabla \eta\rangle G & \leq-2 f t_{1}^{\frac{1}{2}} \frac{|\nabla \eta|}{\eta^{\frac{1}{2}}} G^{\frac{3}{2}} \leq-2 f t_{1} \frac{\sqrt{C_{1}}}{R} G^{\frac{3}{2}} \\
& \leq(1-f) G^{2}+\frac{27 t_{1}^{2}}{16} \frac{C_{1}^{2}}{R^{4}} \frac{f^{4}}{(1-f)^{3}}
\end{aligned}
$$

and

$$
\langle\nabla \log (1-f), \nabla q\rangle \leq|\nabla q| w^{\frac{1}{2}} \leq|q| w+\frac{|\nabla q|^{2}}{4|q|}
$$


Combining the above three inequalities we have

$$
\begin{aligned}
0 \geq & (1-f) G^{2}-2 t_{1} \eta\left(K_{1}+K_{2}-\hat{A}_{f}-\frac{q}{1-f}-\frac{\hat{A}(f)}{1-f}\right) G \\
& -\frac{27 t_{1}^{2}}{16} \frac{C_{1}^{2}}{R^{4}} \frac{f^{4}}{(1-f)^{3}}-\frac{2 t_{1} \eta}{1-f}|q| G-\frac{t_{1}^{2} \eta 2}{2(1-f)} \frac{|\nabla q|^{2}}{|q|} \\
& -\eta G-t_{1}\left(\frac{C_{3}(n)}{R^{2}}+\frac{C_{4}(n)}{R} \sqrt{K_{1}}+C_{2} K_{2}\right) G .
\end{aligned}
$$

From $0 \leq \frac{1}{1-f}<1$ and $0<\frac{-f}{1-f} \leq 1$, we see that

$$
\begin{aligned}
\hat{A}_{f}+\frac{\hat{A}(f)}{1-f} & =\frac{-f}{1-f} \hat{A}_{f}+\frac{1}{1-f}\left(\hat{A}_{f}+\hat{A}(f)\right) \\
& \geq \frac{-f}{1-f} \min \left\{0, \min _{Q_{2 R, T}} \hat{A}_{f}\right\}+\frac{1}{1-f} \min \left\{0, \min _{Q_{2 R, T}} A^{\prime}(u)\right\} \\
& \geq \min \left\{0, \min _{Q_{2 R, T}} \hat{A}_{f}, \min _{Q_{2 R, T}} A^{\prime}(u)\right\} \\
& =-\kappa_{2 R} .
\end{aligned}
$$

By $0<\eta\left(x_{1}, t_{1}\right) \leq 1$, we get

$$
\begin{aligned}
0 \geq & (1-f) G^{2}-G-2 t_{1}\left(K_{1}+K_{2}+\max _{Q_{2 R, T}}(|q|-q)+\kappa_{2 R}\right) G \\
& -t_{1}\left(\frac{C_{3}(n)}{R^{2}}+\frac{C_{4}(n)}{R} \sqrt{K_{1}}+C_{2} K_{2}\right) G-\frac{27 t_{1}^{2}}{16} \frac{C_{1}^{2}}{R^{4}} \frac{f^{4}}{(1-f)^{3}}-\frac{t_{1}^{2}}{2} \max _{Q_{2 R, T}} \frac{|\nabla q|^{2}}{|q|} .
\end{aligned}
$$

Applying the quadratic formula and the inequality of arithmetic and geometric means

$$
\frac{\sqrt{K_{1}}}{R} \leq \frac{1}{2 R^{2}}+\frac{K_{1}}{2}
$$

and noticing the fact $0 \leq \frac{-f}{1-f}<1$ again, we obtain

$$
G \leq C_{6}(n)\left(1+t_{1}\left(\frac{1}{R^{2}}+H\right)\right)
$$

where $C_{6}(n)$ is a constant that depends only on $n$ and

$$
H=K_{1}+K_{2}+\max _{Q_{2 R, T}}(|q|-q)+\max _{Q_{2 R, T}} \frac{|\nabla q|}{\sqrt{|q|}}+\kappa_{2 R} .
$$

Noticing that $d\left(x, x_{0}, T_{1}\right) \leq R$ implies $\eta\left(x, T_{1}\right)=1$, we can get

$$
w\left(x, T_{1}\right)=\frac{F\left(x, T_{1}\right)}{T_{1}} \leq \frac{G\left(x_{1}, t_{1}\right)}{T_{1}} \leq C_{6}(n)\left(\frac{1}{T_{1}}+\frac{1}{R^{2}}+H\right) .
$$

Since $T_{1}$ is arbitrary, and using $\sqrt{x+y} \leq \sqrt{x}+\sqrt{y}$, we complete the proof.

Similar to Corollary 2.6, when $(M, g(0))$ is a complete noncompact Riemannian manifold without boundary and $g(t)$ evolves by (1.2), we can obtain a global estimate from Theorem 3.1 by taking $R \rightarrow 0$.

Corollary 3.5. Let $(M, g(t))$ be a complete solution to (1.2) for $t \in[0, T]$ and $(M, g(0))$ be a complete noncompact Riemannian manifold without boundary. Let $u$ be a positive solution to (1.1). Suppose that there exist constants $L>0$ and $K \geq 0$, such that $u \leq L$ and

Ric $\geq-K_{1} g, \quad h \geq-K_{2} g$. 
Then we have

$$
\frac{|\nabla u(x, t)|}{u(x, t)} \leq \tilde{C}\left(\frac{1}{\sqrt{t}}+\sqrt{H}\right)\left(1+\log \frac{L}{u(x, t)}\right)
$$

on $M \times[0, T]$, where $\tilde{C}$ as in Theorem 3.1 and

$$
H=K_{1}+K_{2}+\sup _{M \times[0, T]}(|q|-q)+\sup _{M \times[0, T]} \frac{|\nabla q|}{\sqrt{|q|}}+\kappa .
$$

The following corollary gives a elliptic Harnack inequality by integrating the elliptic type gradient estimate (3.3) in space only. Unlike Corollary 2.10, this inequality can compare the function values at two spatial points at the same time, but inequality (2.14) cannot.

Corollary 3.6. Let $(M, g(t))$ be a complete solution to (1.2) for $t \in[0, T]$ and $(M, g(0))$ be a complete noncompact Riemannian manifold without boundary. Let $u$ be a positive solution to (1.1). Suppose that there exist constants $L>0$ and $K \geq 0$, such that $u \leq L$ and

$$
\text { Ric } \geq-K_{1} g, \quad h \geq-K_{2} g .
$$

Then for any $x_{1}, x_{2} \in M$, we have

$$
u\left(x_{1}, t\right) \geq u\left(x_{2}, t\right)^{\mathcal{H}} \exp ((1-\mathcal{H})(1+\log L)) .
$$

in each $t \in[0, T]$. Here, $\mathcal{H}=\exp \left(\tilde{C}\left(\frac{1}{\sqrt{t}}+\sqrt{H}\right) d\left(x_{1}, x_{2}, t\right)\right)$, where $\tilde{C}$ as in Theorem 3.1 and

$$
H=K_{1}+K_{2}+\sup _{M \times[0, T]}(|q|-q)+\sup _{M \times[0, T]} \frac{|\nabla q|}{\sqrt{|q|}}+\kappa .
$$

Proof. For any fixed $t$ and any $x_{1}, x_{2} \in M$, let $\zeta:[0,1] \rightarrow M$ is the geodesic of minimal length, which connecting $x_{2}$ and $x_{1}, \zeta(0)=x_{2}$ and $\zeta(1)=x_{1}$. Let $f=\log u$ and

$$
l(s)=\log (1+\log L-f(\zeta(s), t)) .
$$

By Corollary 3.5 we have

$$
\begin{aligned}
\frac{d l(s)}{d s} & =\frac{-\left\langle(\nabla f)(\zeta(s), t), \zeta^{\prime}(s)\right\rangle}{1+\log L-f(\zeta(s), t)} \\
& \leq\left|\zeta^{\prime}(s)\right| \cdot \frac{|\nabla f|(\zeta(s), t)}{1+\log L-f(\zeta(s), t)} \\
& \leq \tilde{C}\left|\zeta^{\prime}(s)\right|\left(\frac{1}{\sqrt{t}}+\sqrt{H}\right) .
\end{aligned}
$$

Integrating this inequality over $\zeta(s)$, we have

$$
\log \frac{1+\log L-f\left(x_{1}, t\right)}{1+\log L-f\left(x_{2}, t\right)} \leq \tilde{C}\left(\frac{1}{\sqrt{t}}+\sqrt{H}\right) d\left(x_{1}, x_{2}, t\right) .
$$

From this inequality, inequality (3.4) can be obtained through a simple calculation.

\section{Applications}

We will give some applications of gradient estimates in section 2 and section 3 to some special equations. In some cases, we also take the geometric flow as the Ricci flow, i.e., $h=-$ Ric in (1.2). 
4.1. Applications of space-time gradient estimates. In this subsection, we focus on applications of space-time gradient estimate for positive solutions. In this case, we see that $\lambda, \Lambda, \Sigma$ are not necessarily finite in (2.12). For example, if we choose $A(u)=u^{p}$ for $p>0$, then $\lambda, \Lambda, \Sigma$ are all multiples of $\sup _{M \times[0, T]} u^{p-1}$ and they are not all zero if $p \neq 1$. But $\sup _{M \times[0, T]} u^{p-1}$ is not necessarily finite, unless $u$ is bounded. For the case that $q=0$ and $A(u)=u^{p}$, the reader can also refer to [20,21,35.

Naturally, for general unbounded positive function $u$, we want to know when $\lambda, \Lambda$ and $\Sigma$ are finite. Since $u$ is a positive solution to (1.1),$A(u)$ can be written as $u \cdot \frac{A(u)}{u}$, which is $A(u)=u H(u)$ for some $C^{2}$ function $H(u)$. As pointed by Q. Chen and the author in [6, Remark 1.8], $\lambda, \Lambda, \Sigma<+\infty$ implies

$$
|H(u)| \leq C_{0} \log u, \quad \text { as } u \rightarrow+\infty .
$$

When $A(u)=a u \log u, \mathrm{~A}$ direct computation yields $\Sigma=0$ and

$$
\begin{cases}\lambda_{2 R}=\lambda \equiv 0, \quad \Lambda_{2 R}=\Lambda \equiv a, & \text { if } a \geq 0, \\ \lambda_{2 R}=\lambda \equiv-a, \quad \Lambda_{2 R}=\Lambda \equiv 0, & \text { if } a \leq 0 .\end{cases}
$$

Therefore, we can obtain that local and global gradient estimates for positive solutions of the equation

$$
\left(\Delta-q(x, t)-\partial_{t}\right) u(x, t)=a u(x, t) \log (u(x, t)), \quad a \in \mathbb{R}
$$

from Theorem 2.1 and Corollary 2.6

Remark 4.1. By the asymptotic behavior of $H$, we can find many examples that satisfy $\lambda, \Lambda, \Sigma<+\infty$. Such as $H(u)=\frac{P_{k}(u)}{P_{l}(u)}$, where $P_{k}, P_{l}$ are polynomials of degree $k, l$, respectively and $k<l$. Hence we can obtain gradient estimates for positive solution of the following series of equations

$$
\left(\Delta-q(x, t)-\partial_{t}\right) u(x, t)=a u(x, t) \frac{P_{k}(u(x, t))}{P_{l}(u(x, t))}, \quad a \in \mathbb{R}, k<l .
$$

For instance, if we choose $k=0, l=1$, so $A(u)=\frac{u}{u+1}$, then $\lambda=\frac{1}{4}, \Lambda=0$ and $\Sigma=\frac{8}{27}$.

On the other hand, as mentioned in Remark 2.9, if $A(u)=a u \log u$, then $\Sigma=0$. In addition, we take $h=-$ Ric. In this case, we don't need the assumption on the bound $|\nabla h|$ since the contracted second Bianchi identity. At this time, when $\alpha \rightarrow 1$, we can also let $\varepsilon \rightarrow 0$ in local estimate (2.1), and then we are arriving at

Corollary 4.2. Let $(M, g(0))$ be a complete Riemannian manifold, and let $g(t)$ evolves by the Ricci flow for $t \in[0, T]$. Suppose that there exist constants $K_{2}, \theta \geq 0$ such that

$$
0 \leq \operatorname{Ric} \leq K_{2} g
$$

and

$$
\Delta q \leq \theta_{2 R}
$$

on $Q_{2 R, T}$. If $u$ is a positive solution to (4.1). Then on $Q_{R, T}$, we have

(1) for $a \geq 0$,

$$
\frac{|\nabla u(x, t)|^{2}}{u^{2}(x, t)}-\frac{u_{t}(x, t)}{u(x, t)}-q(x, t)-\frac{A(u(x, t))}{u(x, t)} \leq \frac{n}{t}+(C+n) K_{2}+\sqrt{n \theta}+\frac{n}{2} a
$$

(2) for $a<0$,

$$
\frac{|\nabla u(x, t)|^{2}}{u^{2}(x, t)}-\frac{u_{t}(x, t)}{u(x, t)}-q(x, t)-\frac{A(u(x, t))}{u(x, t)} \leq \frac{n}{t}+(C+n) K_{2}+\sqrt{n \theta}-n a,
$$

where $C$ as in Theorem 2.1.

From the above local estimate, we have immediately 
Corollary 4.3. Let $(M, g(0))$ be a complete noncompact Riemannian manifold without boundary, and let $g(t)$ evolves by the Ricci flow for $t \in[0, T]$. Suppose that there exist constants $K_{2}, \theta \geq 0$ such that

$$
0 \leq \text { Ric } \leq K_{2} g
$$

and

$$
\Delta q \leq \theta .
$$

If $u$ is a positive solution to (4.1). Then we have

$$
\frac{|\nabla u(x, t)|^{2}}{u^{2}(x, t)}-\frac{u_{t}(x, t)}{u(x, t)}-q(x, t)-\frac{A(u(x, t))}{u(x, t)} \leq \frac{n}{t}+C^{\prime \prime}\left(K_{2}+\sqrt{\theta}+|a|\right)
$$

on $M \times[0, T]$, where $C^{\prime \prime}$ is a constant that depends only on $n$.

When the manifold is closed, we also have

Corollary 4.4. Let $(M, g(t))$ be a closed Riemannian manifold, where $g(t)$ evolves by the Ricci flow for $t \in[0, T]$ and satisfies

$$
0 \leq \operatorname{Ric} \leq K_{2} g .
$$

If $u$ is a positive solution to the equation

$$
\left(\Delta-q(x, t)-\partial_{t}\right) u(x, t)=a u(x, t) \log (u(x, t)),
$$

and $q(x, t)$ satisfies

$$
\Delta q \leq \theta
$$

Then we have

$$
\frac{|\nabla u(x, t)|^{2}}{u^{2}(x, t)}-\frac{u_{t}(x, t)}{u(x, t)}-q(x, t)-\frac{A(u(x, t))}{u(x, t)} \leq \frac{n}{2 t}+n K_{2}+\sqrt{n \theta}+\frac{n}{2}|a|
$$

on $M \times(0, T]$.

4.2. Applications of elliptic type gradient estimates. Now we give some applications of elliptic type gradient estimates for bounded positive solutions. Since we are dealing with bounded positive solutions, $A(u)$ that satisfies the conditions $\kappa<+\infty$ is easy to find.

We will consider that elliptic type gradient estimates for bounded positive solutions of the equation

$$
\left(\Delta-q(x, t)-\partial_{t}\right) u(x, t)=a u(x, t)^{\beta}, \quad a \in \mathbb{R}, \beta \in(-\infty, 0] \cup[1,+\infty) .
$$

In order not to be redundant, we only give the global estimate here, and the local one is omitted.

Corollary 4.5. Let $(M, g(t))$ be a complete solution to (1.2) for $t \in[0, T]$ and $(M, g(0))$ be a complete noncompact Riemannian manifold without boundary. Let $u$ be a positive solution to (4.2). Suppose that there exist constants $L>0$ and $K \geq 0$, such that $u \leq L$ and

$$
\text { Ric } \geq-K_{1} g, \quad h \geq-K_{2} g .
$$

Then on $M \times[0, T]$, we have

$$
\frac{|\nabla u(x, t)|}{u(x, t)} \leq \tilde{C}\left(\frac{1}{\sqrt{t}}+\sqrt{H^{\prime}}\right)\left(1+\log \frac{L}{u(x, t)}\right),
$$

where $\tilde{C}$ as in Theorem 3.1 and

$$
H^{\prime}=K_{1}+K_{2}+\sup _{M \times[0, T]}(|q|-q)+\sup _{M \times[0, T]} \frac{|\nabla q|}{\sqrt{|q|}}+\kappa_{0}
$$

with

$$
\kappa_{0}= \begin{cases}\frac{\operatorname{sign} a-1}{2} a \beta L^{\beta-1}, & \text { if } a \in \mathbb{R}, \beta \geq 1, \\ 0, & \text { if } a \leq 0, \beta \leq 0, \\ a(1-\beta)\left(\inf _{M \times[0, T]} u(x, t)\right)^{\beta-1}, & \text { if } a \geq 0, \beta \leq 0 .\end{cases}
$$

Here, $\operatorname{sign} a$ is the sign function, which is $1,0,-1$ if $a>0,=0,<0$, respectively. 
Proof. From Corollary 3.5, we just have to compute $\kappa$. By the definition, we have

$$
\begin{aligned}
\kappa & =-\min \left\{0, \inf _{M \times[0, T]}\left(a \beta u^{\beta-1}\right), \inf _{M \times[0, T]}\left(a(\beta-1) u^{\beta-1}\right)\right\} \\
& = \begin{cases}0, & \text { if } a \geq 0, \beta \geq 1, \\
-a \beta L^{\beta-1}, & \text { if } a \leq 0, \beta \geq 1, \\
0, & \text { if } a \leq 0, \beta \leq 0, \\
a(1-\beta)\left(\inf _{M \times[0, T]} u(x, t)\right)^{\beta-1}, & \text { if } a \geq 0, \beta \leq 0 .\end{cases}
\end{aligned}
$$

Therefore, we obtain the corollary.

In particular, when $q(x, t)=$ const., the term $q u(x, t)$ can be combined by $a u(x, t)^{\beta}$, so we get

Corollary 4.6. Let $(M, g(t))$ be a complete solution to (1.2) for $t \in[0, T]$ and $(M, g(0))$ be a complete noncompact Riemannian manifold without boundary. Let $u$ be a positive solution to

$$
\left(\Delta-\partial_{t}\right) u(x, t)=a u(x, t)^{\beta}, \quad a \in \mathbb{R}, \beta \in(-\infty, 0] \cup[1,+\infty) .
$$

Suppose that there exist constants $L>0$ and $K \geq 0$, such that $u \leq L$ and

$$
\text { Ric } \geq-K_{1} g, \quad h \geq-K_{2} g .
$$

Then on $M \times[0, T]$, we have

$$
\frac{|\nabla u(x, t)|}{u(x, t)} \leq \tilde{C}\left(\frac{1}{\sqrt{t}}+\sqrt{K_{1}+K_{2}+\kappa_{0}}\right)\left(1+\log \frac{L}{u(x, t)}\right)
$$

where $\tilde{C}$ as in Theorem 3.1 and

$$
\kappa_{0}= \begin{cases}\frac{\operatorname{sign} a-1}{2} a \beta L^{\beta-1}, & \text { if } a \in \mathbb{R}, \beta \geq 1, \\ 0, & \text { if } a \leq 0, \beta \leq 0, \\ a(1-\beta)\left(\inf _{M \times[0, T]} u(x, t)\right)^{\beta-1}, & \text { if } a \geq 0, \beta \leq 0 .\end{cases}
$$

Here, $\operatorname{sign} a$ is the sign function, which is $1,0,-1$ if $a>0,=0,<0$, respectively.

Remark 4.7. For each of these specific equations that appear in this section, we also have the corresponding Harnack inequality, which we will not write them all down here.

\section{REFERENCES}

[1] M. Bailesteanu, X. Cao, and A. Pulemotov. Gradient estimates for the heat equation under the Ricci flow. Journal of Functional Analysis, 258(10):3517-3542, 2010.

[2] E. Calabi. An extension of E. Hopfs maximum principle with an application to Riemannian geometry. Duke Mathematical Journal, 25(1):45-56, 1958.

[3] X. Cao and R. S. Hamilton. Differential Harnack estimates for time-dependent heat equations with potentials. Geometric and Functional Analysis, 19(4):989-1000, 2009.

[4] L. Chen and W. Chen. Gradient estimates for a nonlinear parabolic equation on complete non-compact Riemannian manifolds. Annals of Global Analysis and Geometry, 35(4):397-404, 2009.

[5] Q. Chen and H. Qiu. Gradient estimates and Harnack inequalities of a nonlinear parabolic equation for the $V$ Laplacian. Annals of Global Analysis and Geometry, 50(1):47-64, 2016.

[6] Q. Chen and G. Zhao. Li-Yau type and Souplet-Zhang type radient estimates of a parabolic equation for the $V$-laplacian. Journal of Mathematical Analysis and Applications, 463(2):744-759, 2018.

[7] S. Y. Cheng and S.-T. Yau. Differential equations on Riemannian manifolds and their geometric applications. Communications on Pure and Applied Mathematics, 28(3):333-354, 1975.

[8] B. Chow, P. Lu, and L. Ni. Hamilton's Ricci flow, volume Graduate Studies in Mathematics 77. American Mathematical Society, 2006.

[9] N. T. Dung and N. N. Khanh. Gradient estimates of Hamilton-Souplet-Zhang type for a general heat equation on Riemannian manifolds. Archiv der Mathematik, 105(5):479-490, 2015.

[10] C. M. Guenther. The fundamental solution on manifolds with time-dependent metrics. Journal of Geometric Analysis, 12(3):425-436, 2002.

[11] H. Guo and M. Ishida. Harnack estimates for nonlinear backward heat equations in geometric flows. Journal of Functional Analysis, 267(8):2638-2662, 2014.

[12] R. S. Hamilton. Three-maniflods with positive Ricci flow. Journal of Differential Geometry, 1:255-306, 1982. 
[13] R. S. Hamilton. The Harnack estimate for the Ricci flow. Journal of Differential Geometry, 37:225-243, 1993.

[14] R. S. Hamilton. A matrix Harnack estimate for the heat equation. Communications in Analysis and Geometry, 1(1):113-126, 1993.

[15] R. S. Hamilton. Harnack estimate for the mean curvature flow. Journal of Differential Geometry, 41:215-226, 1995.

[16] M. Ishida. Geometric flows and differential Harnack estimates for heat equations with potentials. Annals of Global Analysis and Geometry, 45(4):287-302, 2014.

[17] J. Li. Gradient estimate for the heat kernel of a complete Riemannian manifold and its applications. Journal of Functional Analysis, 97(2):293-310, 1991.

[18] P. Li and S.-T. Yau. On the parabolic kernel of the Schrödinger operator. Acta Mathematica, 156(1):153-201, 1986.

[19] Y. Li. Li-Yau-Hamilton estimates and Bakry-Emery-Ricci curvature. Nonlinear Analysis: Theory, Methods 83 Applications, 113:1-32, 2015.

[20] Y. Li and X. Zhu. Li-Yau Harnack estimates for a heat-type equation under the geometric flow. Potential Analysis, 2018. https://doi.org/10.1007/s11118-018-9739-x

[21] Y. Li and X. Zhu. Harnack estimates for a heat-type equation under the Ricci flow. Journal of Differential Equations, 260(4):3270-3301, 2016.

[22] S. Liu. Gradient estimates for solutions of the heat equation under Ricci flow. Pacific Journal of Mathematics, 243(1):165-180, 2009.

[23] L. Ma. Gradient estimates for a simple elliptic equation on complete non-compact Riemannian manifolds. Journal of Functional Analysis, 241(1):374-382, 2006

[24] E. R. Negrin. Gradient estimates and a Liouville type theorem for the Schrödinger operator. Journal of Functional Analysis, 127(1):198-203, 1995.

[25] L. Ni. Ricci flow and nonnegativity of sectional curvature. Mathematical Research Letters, 11(6):883-904, 2004.

[26] F. Rothe. Global Solutions of Reaction-Diffusion Systems. Springer, 1984.

[27] Q. Ruan. Elliptic-type gradient estimate for Schrödinger equations on noncompact manifolds. Bulletin of the London Mathematical Society, 39(6):982-988, 2007.

[28] J. Smoller. Shock Waves and Reaction-Diffusion Equations. Springer-Verlag, 1983.

[29] P. Souplet and Q. S. Zhang. Sharp gradient estimate and Yau's Liouville theorem for the heat equation on noncompact manifolds. Bulletin of the London Mathematical Society, 38(6):1045-1053, 2006.

[30] J. Sun. Gradient estimates for positive solutions of the heat equation under geometric flow. Pacific Journal of Mathematics, 253(2):489-510, 2011.

[31] J.-Y. Wu. Elliptic gradient estimates for a weighted heat equation and applications. Mathematische Zeitschrift, 280(1-2):451-468, 2015.

[32] J.-Y. Wu. Elliptic gradient estimates for a nonlinear heat equation and applications. Nonlinear Analysis: Theory, Methods 83 Applications, 151:1-17, 2017.

[33] Y. Yang. Gradient estimates for a nonlinear parabolic equation on Riemannian manifolds. Proceedings of the American Mathematical Society, 136(11):4095-4102, 2008.

[34] Q. S. Zhang. Some gradient estimates for the heat equation on domains and for an equation by Perelman. International Mathematics Research Notices, 2006(1):1479-1485, 2006.

[35] L. Zhao and S. Fang. Gradient estimates for a nonlinear Lichnerowicz equation under general geometric flow on complete noncompact manifolds. Pacific Journal of Mathematics, 285(1):243-256, 2006.

Guangwen Zhao, School of Mathematical Sciences, Fudan University, Shanghai 200433, China

E-mail address: gwzhao@fudan.edu.cn 